\title{
Eine Systematik der Forschungsformen und ihre Eignung für Forschendes Lernen ${ }^{1}$
}

\section{Harald A. Mieg}

Forschen nutzt die ganze Vielfalt von Erkenntnismöglichkeiten - genaues Beobachten ist ebenso wichtig wie Hochtechnologie. In diesem Beitrag werden verschiedene Forschungsformen im Hinblick auf Erkenntnisweisen systematisiert und Wege für die differenzielle Nutzung der Forschungsformen für das Forschende Lernen aufgezeigt.

\section{$1 \quad$ Einleitung}

Wie wirkt sich unser Forschungsverständnis auf die Umsetzung Forschenden Lernens aus? Ist eine Rechnersimulation in gleicher Weise geeignet, forschend in Wissenschaft einzuführen, wie eine Analyse historischer Quellen? Mieg und Dinter gingen dieser Frage nach, indem sie eine Forschungs-Typologie des Wissenschaftsrats (2012) auf Forschendes Lernen übertrugen (Mieg \& Dinter 2017). Mein Beitrag dient darauf aufbauend zwei Zielen:

1 Ich danke den Kollegen Ludwig Huber und Hubert Laitko für ihre Kommentare zu einer Vorfassung dieses Kapitels. 
- Erstens soll die Forschungstypologie in eine systematische Form gebracht werden (Kapitel 2).

- Zweitens möchte ich die Auswirkungen von unterschiedlichen Forschungsformen auf Forschendes Lernen an neueren Befunden überprüfen und ein hierzu ein Fazit ziehen (Kapitel 3).

\section{Systematik}

Einen Überblick über die Forschungsformen, die für Forschendes Lernen relevant sein können, gibt Tabelle 1 (Mieg \& Dinter, 2017). Diese Forschungsformen werden im Folgenden systematisch charakterisiert - zum einen mit Blick auf unterschiedliche Forschungstätigkeiten (2.1), zum anderen hinsichtlich der damit verbundenen Erkenntnisweisen (2.2).

\subsection{Forschungstätigkeiten}

Forschen ist in erster Hinsicht eine Tätigkeit bzw. ein Bündel von Tätigkeiten (siehe auch Reinmann, 2018). Diese Forschungstätigkeiten lassen sich anhand von vier Grundtätigkeiten des Forschens unterscheiden:

1. Beobachten: von der Naturbeobachtung (in der Astronomie oder Biologie) oder der teilnehmenden Beobachtung bis hin zur systematischen Erhebung großer Datenmengen;

2. Beurteilen: vom Interpretieren von Texten und Deuten von Bildern bis hin zur formalen Theoriebildung;

3. Modellieren: von der Darstellung guter Praxis (seit jeher in Medizin und Ingenieurwesen) bis hin zu rechnergestützten Großmodellen (in Volkswirtschaftslehre oder Klimaforschung);

4. Konstruieren: von der technischen Entwicklung bis zum künstlerischen Gestalten.

Die Vorstellung der Systematik in Tabelle 1 beginnt mit Beispielen für Methoden (z. B. Interview) und Einrichtungen (z. B. Labore) und soll eine erste Idee von den unterschiedlichen Forschungsformen vermitteln. Grundsätzlich anzumerken ist:

- Die Systematik - insbesondere die der Forschungstätigkeiten - ist als pragmatisch hinsichtlich des verfolgten Zwecks zu verstehen. Die ursprüngliche Typologie 


\begin{tabular}{|c|c|c|c|c|c|}
\hline 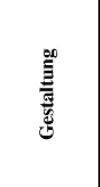 & 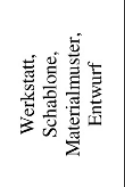 & 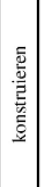 & 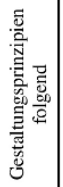 & 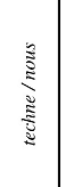 & 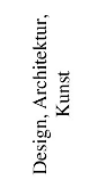 \\
\hline 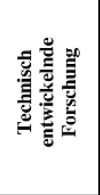 & 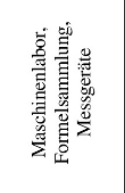 & 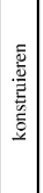 & & 气ूँ & 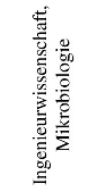 \\
\hline 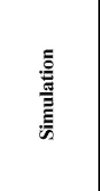 & 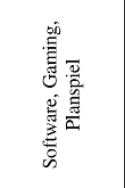 & 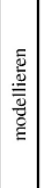 & 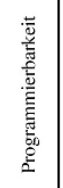 & 气ूँ & 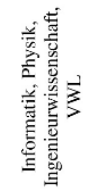 \\
\hline 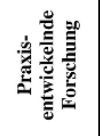 & 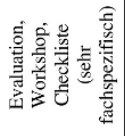 & 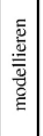 & & 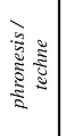 & 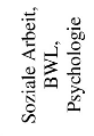 \\
\hline 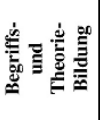 & 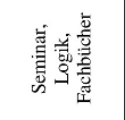 & 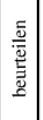 & 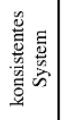 & 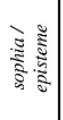 & 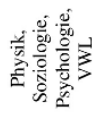 \\
\hline 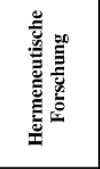 & 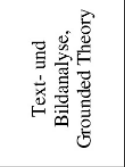 & 흘 & & 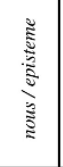 & 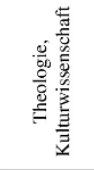 \\
\hline 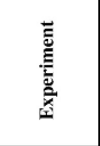 & 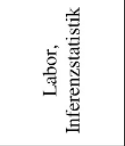 & 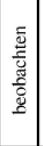 & 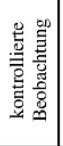 & 气े 气ू & 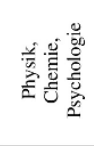 \\
\hline 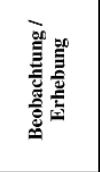 & 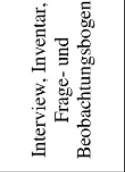 & 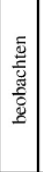 & & 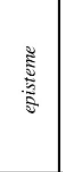 & 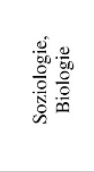 \\
\hline 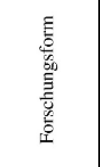 & 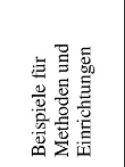 & 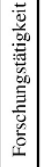 & 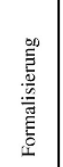 & 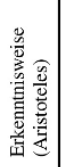 & 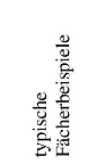 \\
\hline
\end{tabular}


durch den Wissenschaftsrat (2012) bezog sich auf Formen der Datenproduktion und -sicherung. Der Kontext hier ist Forschendes Lernen. Eine Herleitung und wissenschaftstheoretische Diskussion der Forschungsformen ist bei Mieg und Dinter zu finden (Mieg \& Dinter, 2017).

- Die Darstellung der Forschungsformen entspricht einem gängigen Verständnis, wobei jede Forschungsform auch in der Wissenschaft unterschiedliche Nutzungsund Interpretationsweisen kennt. So wird der Begriff des Experiments hier eng gefasst, so, wie er sich seit Bacon in der Physik entwickelt hat (z. B. Sagasti, 2000).

- Neu an der Typologie durch den Wissenschaftsrat war die Vorstellung von Begriffs- und Theoriebildung als eigener Form der Forschung. Üblicherweise wird Theoriebildung als Teil eines umfassenden Erkenntnisprozesses verstanden. Neu an der hier vorgestellten Systematik ist - neben dem Bezug zu Forschungstätigkeiten und Erkenntnisweisen - die Einführung von ,praxis-entwickelnder Forschung" als eigener Forschungsform.

In Tabelle 1 werden die acht Forschungsformen als Paare vorgestellt und Grundtätigkeiten des Forschens zugeordnet (dritte Zeile). Die jeweils zweite Forschungsform stellt hierbei eine weitergehende Formalisierung, das heißt eine methodische oder begriffliche „Zuspitzung“ dar (Mieg, de Sombre \& Naef, 2013):

- Beobachtung/Erhebung und Experiment (forschende Grundtätigkeit: „beobachten"): Beobachtung/Erhebung meint eine methodische und dokumentierte Beobachtung, wie sie schon früh in der Astronomie oder der Tier- und Pflanzenkunde genutzt wurde. Das Experiment im engeren Sinne ist eine formalisierte Beobachtung: Unter kontrollierten Bedingungen werden Effekte erzeugt (von Wright, 1984).

- Hermeneutische Forschung und Begriffs- und Theorie-Bildung („beurteilen“): Hermeneutik bezieht sich zuerst auf die Auslegung von Texten, zum Beispiel von Bibel- oder Rechtstexten. In einem erweiterten Sinn wird Hermeneutik zum Verstehen von soziokulturellen Kontexten genutzt. Begriffs- und Theoriebildung zielt darüber hinaus auf ein konsistentes, das heißt widerspruchsfreies System von Annahmen (z. B. Bunge, 1967).

- Praxisentwickelnde Forschung und Simulation („modellieren“): Praxisentwickelnde Forschung modelliert Vorgänge, wie sie sind oder sein sollen (z. B. Ablaufschema für konfliktfreie Gespräche mit gewaltbereiten Jugendlichen). Sie bietet praktisch nutzbare Abbildungen der Welt. Simulation stellt eine Verschärfung dar, indem Programmierbarkeit unterstellt wird. Simulation, so komplex sie aussehen mag, ist immer eine verkürzte Weltdarstellung (Mieg, 1993). 
- Technisch entwickelnde Forschung und Gestaltung („konstruieren“): Technisch entwickelnde Forschung ist funktionales Konstruieren mit Bezug auf ein Problem, das es zu lösen gilt (zu finden im Maschinenbau). Gestaltung ist Konstruieren mit Blick auf ästhetisch-funktionale Gestaltungsprinzipien (zu finden im Design oder in der Architektur, z. B. Alexander, 1964).

\subsection{Weisen der Erkenntnis durch Forschung}

Forschen (beobachten, erkunden, nachforschen, testen ...) nutzt die ganze Vielfalt der Erkenntnis. Bereits Aristoteles unterschied Wissenschaft in einem engen Sinne (Theorien) von den vielfältigen Wegen der Erkenntnisgewinnung. Er benannte fünf Weisen der theoretischen und praktischen Erkenntnis, „Mittel, mit denen die Seele bejahend oder verneinend die Wahrheit trifft" (Aristoteles, 2007, S. 241). Diese werden hier in Zusammenhang mit den Forschungsformen vorgestellt (Tab. 1, fünfte Zeile): ${ }^{2}$

1. episteme (Wissen, Wissenschaft): Forschende Wissenschaft beginnt mit empirischer Beobachtung (Forschungsform „Beobachtung/Erhebung").

2. sophia (Theorie, Weisheit): Höchste wissenschaftliche Erkenntnis im engeren Sinne ist Theoriebildung („Begriffs- und Theoriebildung“).

3. techne (Kunst, Können): Etwas gezielt funktionsfähig zu machen - zum Beispiel eine Maschine zu bauen - bedeutet auch Erkenntnis, und zwar Funktionserkenntnis (z. B. „Technisch entwickelnde Forschung“).

4. phronesis (Klugheit, praktisches Verständnis): In wertebestimmten Zusammenhängen - wie Politik oder Berufsalltag - das mögliche Vorgehen aufzuzeigen ist eine eigene Weise von Erkenntnis („Praxis-entwickelnde Forschung“).

5. nous (Vernunft, Geist, Intuition): Gemeint ist hier das Verstehen als eigene Erkenntnisweise (von Wright, 1984), so in der hermeneutischen Forschung.

Forschungsformen und Erkenntnisweisen sind einander nicht eindeutig zugeordnet. Die ersten vier Forschungsformen können auch in einem weiten Sinne zu wissenschaftlicher Erkenntnis (episteme) zählen. Beim Experiment kommt zudem Kunstfertigkeit (techne) ins Spiel. Die vier letzten Forschungsformen beziehen sich

2 Um den Zusammenhang klarer darzustellen habe ich die Reihenfolge geändert und die Erkenntnisweisen spezifisch interpretiert. Meines Erachtens bleibe ich damit näher an Aristoteles' Wissenschaftsverständnis als z. B. Flyvbjerg (2001), der Sozialwissenschaften generell durch phronesis charakterisiert. 
im weitesten Sinn alle auf Erkenntnis durch Kunstfertigkeit (techne). ${ }^{3}$ Das griechische techne entspricht nicht ganz dem deutschen Verständnis von Kunst oder Technik: techne setzt nach Aristoteles Ursachenkenntnis voraus, ist ein rationales Seelenvermögen und somit erkenntnisrelevant (Ritter \& Gründer, 1998).

\section{Forschungsformen und Forschendes Lernen}

Welche Relevanz haben die Forschungsformen für das Forschende Lernen? Eignen sich die Forschungsformen in gleicher Weise zum Einstieg in Forschendes Lernen? Mieg und Dinter prüften diese Fragen (Mieg \& Dinter, 2017) anhand von zwei Referenzwerken zum Forschenden Lernen: der programmatischen Schrift der BAK (BAK, 1970) sowie dem neueren Übersichtsband von Mieg und Lehmann (Mieg \& Lehmann 2017). Diese erste Einschätzung wird im Folgenden anhand der überarbeiteten Systematik der Forschungsformen (Tab. 1) und aktueller Literatur noch einmal überprüft; dabei wird für jede Forschungsform ein Fazit gezogen. Tabelle 2 vermittelt hierzu den Überblick.

Grundsätzlich kann unterschieden werden zwischen Forschungsformen, die einen einfachen Einstieg in Forschendes Lernen (z. B. Beobachtung/Erhebung, in Tab. 2 als „einfach“ kategorisiert) oder einen schwierigen Einstieg (z. B. Begriffsund Theorie-Bildung, in Tab. 2 als „schwierig“ kategorisiert) darstellen. Es gibt allerdings auch Forschungsformen, bei denen zusätzliche Voraussetzungen diese allgemeine Einteilung erweitern. Praxisentwickelnde Forschung oder Gestaltung sind bei Vorliegen gewisser Vorkenntnisse als "nicht schwierig“ anzusehen, Forschungsformen, bei denen methodische Randbedingungen oder Laborzugänge notwendig sind, können dagegen im Hinblick auf ihre Eignung für Forschendes Lernen als „aufwendig“ klassifiziert werden.

3 Die in Tab. 1 vorgestellte Systematik bringt Gestaltung in einen Zusammenhang mit hermeneutischer Forschung. Der Zusammenhang besteht über die Zuordnung zu derselben Erkenntnisform (nous). Dies entspricht z. B. dem semiotischen Ansatz der Arbeiten von Alexander (1964) für die Architektur oder Monö (1997) für das Fach Design. Forschendes Lernen in der Kunst wird bei Kaufmann, Satilmis \& Mieg (2018) den hermeneutischen Ansätzen zugerechnet. 


\begin{tabular}{|c|c|c|c|c|}
\hline 总 & 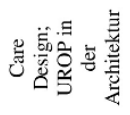 & & & 点高 \\
\hline 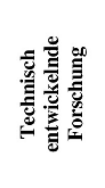 & 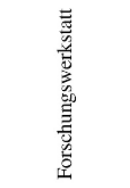 & 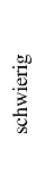 & 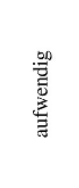 & 㠿 \\
\hline 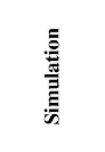 & 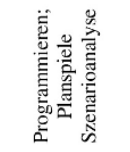 & 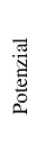 & $\begin{array}{l}\text { 总 } \\
\text {. }\end{array}$ & $\begin{array}{l}\text { 苞 } \\
\text {. }\end{array}$ \\
\hline 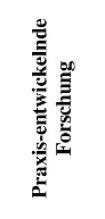 & 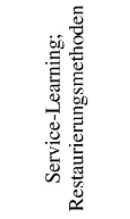 & . & ' & 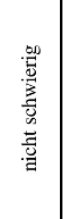 \\
\hline 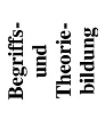 & 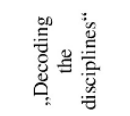 & 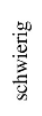 & 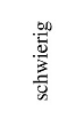 & 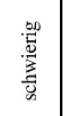 \\
\hline 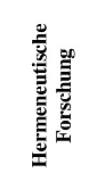 & 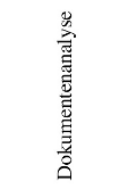 & 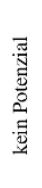 & 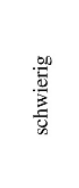 & 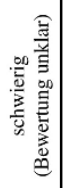 \\
\hline 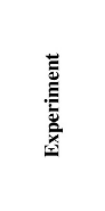 & 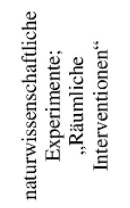 & 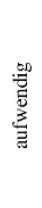 & 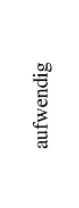 & 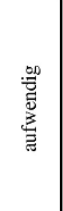 \\
\hline 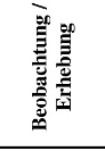 & 营产 & $\begin{array}{l}\text { 总 } \\
\text { : }\end{array}$ & $\begin{array}{l}\text { 总 } \\
\text {. } \\
\text {. }\end{array}$ & 总 \\
\hline & 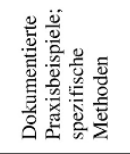 & 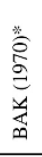 & 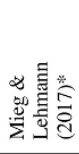 & 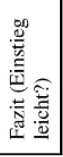 \\
\hline
\end{tabular}


1. Beobachtung/Erhebung: Die Beobachtung und das Erheben von Daten, zum Beispiel mittels Interviews oder Fragebögen, spielen im Forschenden Lernen eine wichtige Rolle. In mehr als einem Drittel der Beiträge im Praxisbuch von Lehmann und Mieg (2018) wird berichtet, dass Studierende Befragungen durchführen oder systematische Beobachtungen - auch Selbstbeobachtungen - vornehmen. Schon die BAK-Schrift zum Forschenden Lernen (BAK, 1970) verwies darauf, dass Beobachtungen vor Ort - sei es mittels sozialwissenschaftlicher Befragung, sei es im klinischen „Unterricht am Krankenbett“ (BAK, 1970, S. 40) - einen leichten Einstieg in Forschung erlauben: Die Studierenden können „mal loslegen“. Beobachtung/Erhebung wird oft in Kombination mit anderen Methoden oder Forschungsformen genutzt. So können Lehramtsstudierende zu „Bildungsforschern“ werden: Die Studierenden entwickelten Fragebögen und nutzten diese für eine experimentelle Interventionsstudie im Biologieunterricht (Schwanewedel, Großschedl \& Heyduck, 2018). Um eine systematische, fachgerechte Einübung in Beobachtung/Erhebung zu gewährleisten, wurde an der Fachhochschule Potsdam für den Bereich Soziale Arbeit das „Werkstatt“-Konzept entwickelt (Schmidt-Wenzel \& Rubel, 2018).

Fazit: Beobachtung/Erhebung bietet für Studierende einen einfachen, ersten Einstieg in Forschung und wird für Forschendes Lernen vielfältig genutzt.

2. Experiment: Das Experiment im engeren Sinne als kontrollierte Beobachtung ist ein unverzichtbarer Bestandteil naturwissenschaftlicher Forschung und wurde im 19. Jahrhundert von der Psychologie übernommen. Die BAK-Schrift (BAK, 1970) sieht curriculare Probleme für Forschendes Lernen mit Experimenten, Mieg und Dinter verweisen zudem auf hohe Laborkosten (Mieg \& Dinter, 2017, S. 37 f.). Labore sind in der Regel teuer und nur begrenzt verfügbar; das gilt auch heute noch. Das Projekt „Humboldt reloaded“ macht deutlich, wie in den Lebenswissenschaften Experimentieren für Forschendes Lernen genutzt werden kann (Selje-Aßmann et al., 2017). Darüber hinaus ist ein mehr oder weniger kontrolliertes Beobachten - Experimentieren in weiteren Sinne von Ausprobieren - immer möglich, zum Beispiel in Form von „räumlichen Interventionen“, wie Albrecht und Ruhl sie schildern: Räume an der Hochschule werden gezielt verändert, um die Wirkung auf das Lernen zu beobachten (Albrecht \& Ruhl, 2018). Fazit: Die Nutzung des Experiments als Forschungsform kann wegen der nötigen Labore aufwendig sein, ist aber in den Naturwissenschaften unverzichtbar.

3. Hermeneutische Forschung: Der hermeneutischen Forschung wird in der BAKSchrift (BAK, 1970) geringes Potenzial für Forschendes Lernen zugesprochen. Inzwischen gibt es jedoch schöne Beispiele, etwa interdisziplinäre Projekte mit historischer Fragestellung, die im Rahmen von InterFlex an der Fachhochschule Potsdam durchgeführt wurden, so zur Migrationsgeschichte oder zu DDR-Ge- 
fangenenlagern (Freund, 2013). 2018 ist ein Band erschienen, der sich eigens mit Hermeneutik im Forschenden Lernen befasst (Kaufmann, Satilmis \& Mieg, 2018). Das Beispiel der historischen Forschung zeigt die Schwierigkeit der Nutzung von Hermeneutik für Forschendes Lernen. Es ist durchaus einfach, Interviews, beispielsweise mit Zeitzeugen, zu führen. Die systematische Interpretation, etwa über ein Verfahren wie die Grounded Theory, ist aber weniger trivial. Hierzu braucht es methodische Kenntnis und Erfahrung (Rubel, 2017).

Fazit: Wenn es um Forschendes Lernen als selbstständiges Forschen - auch bei Bachelor-Studierenden - geht, so bleibt meines Erachtens der Einstieg in die Forschung bei der hermeneutischen Forschungsform eher schwierig.

4. Begriffs- und Theorieentwicklung: In der BAK-Schrift wird argumentiert, dass wegen der hohen Theorieansprüche in vielen Disziplinen Forschendes Lernen mit Begriffs- und Theorieentwicklung nur eingeschränkt und bestenfalls bezogen auf „Teilsysteme“ (BAK, 1970, S. 50) möglich ist. Dennoch finden wir in der neuen Literatur einige Ansätze: Dargestellt wird zum Beispiel, wie Studierende der Betriebswirtschaftlehre mittels „Systemaufstellungen“ Hypothesen- und Konzeptbildung durchführen (Müller-Christ, 2018). Die Anleitung bedarf jedoch beträchtlicher Coaching-Kompetenzen auf Seite der Lehrenden. Vielversprechend ist auch die Methode des "Decoding the disciplines" (Pace \& Middendorf, 2004). In der praktischen Anwendung geht es beispielsweise um die Anleitung der Studierenden zum wissenschaftlichen Schreiben (Kaduk \& Lahm, 2018). Fazit: Begriffs- und Theoriebildung scheint als Forschungsform - zumindest für studentische Forschung im Bachelorstudium - schwierig, da die Theorieansprüche oder die Anforderungen an die Betreuung hoch sind.

5. Praxis-entwickelnde Forschung: Forschendes Lernen mit Praxis-entwickelnder Forschung ist seit den 1990er-Jahren in der Umwelt- und Planungsforschung etabliert, erfolgt dort jedoch unter dem Titel der Transdisziplinarität; beispielhaft sind Projekte zur städtischen Transformation (Prytula, Schröder \& Mieg, 2017). Praxis-entwickelnde Forschung ist zudem gängig in der Restaurierung (z. B. Birresborn, 2017) und findet sich in vielen Projekten Forschenden Lernens, die expliziten Anwendungsbezug haben: So kann Service Learning - das heißt bürgerschaftliches Engagement - beforscht und zugleich eingeübt werden (Mehling, Tienes \& Schuchardt 2018). Damit entwickelt sich nicht zuletzt die Beziehung zwischen einer Hochschule und externen Partnern.

Anzumerken bleibt, dass für Praxis-entwickelnde Forschung gewisse professionelle Vorerfahrung im Feld hilfreich ist. So wird an der Fachhochschule Potsdam der Studiengang Urbane Zukunft, der dem Forschenden Lernen verpflichtet ist (Prytula \& Schröder, 2018), nur als Masterstudiengang angeboten. 
Fazit: Praxis-entwickelnde Forschung ist als Forschungsform für Forschendes Lernen geeignet und nicht schwierig, sofern das Praxisverständnis bei den Studierenden gegeben ist.

6. Simulation: In der BAK-Schrift (BAK, 1970) wird der Simulation ausdrücklich Potenzial für Forschendes Lernen zugeschrieben (Mieg \& Dinter, 2017, S. 38 f.). Durch die enorme Entwicklung der Computer und des Internets ist Simulation durch Programmierung tatsächlich eine leicht nutzbare Forschungsform für Forschendes Lernen geworden. So wird für die Projektlabore der Technischen Universität Berlin unter anderem die Internet-Programmiersprache Python verwendet (Born \& Bor, 2018, S. 175). Simulation mittels Programmierung kommt insbesondere zum Einsatz, wenn Experimente zu aufwendig wären (Mieg \& Lehmann, 2017, z. B. S. 237 f.).

Die BAK-Schrift (BAK, 1970) nennt Planspiele als Beispiel für Forschendes Lernen mit Simulation. Umgesetzt wurde ein Planspiel beispielsweise zu Problemen des nachhaltigen Landschaftsmanagements bei der lokalen Bioenergie- und Nahrungsproduktion in Nordwest-Vietnam (Lippe, 2018). Im Umwelt- und Planungsbereich unterstützen zudem Szenarioanalysen das Forschende Lernen (Prytula, Schröder \& Mieg, 2017).

Fazit: Simulation, insbesondere durch Programmierung, ist aus den Wissenschaften nicht mehr fortzudenken und bietet für Studierende einen einfachen Einstieg in Forschung.

7. Technisch entwickelnde Forschung: Gerade Studierende der Ingenieurwissenschaften können von Anfang an eigene Forschungsprojekte ausführen (Lehmann \& Mieg, 2018), zum Beispiel bei der Entwicklung von Bremssystemen für Fahrräder (Radermacher, Runge \& Scherfner, 2018).

Mieg und Dinter sprechen in diesem Zusammenhang von einem „Paradigmenwechsel“ (Mieg \& Dinter, 2017, S. 38) von der Vorstellung, dass Studierende der technischen Fächer erst einmal über ein gefestigtes theoretisches Grundlagenwissen verfügen müssen (noch in der BAK-Schrift, 1970), hin zum Ansatz, sich Wissen mit ersten eigenen Projekten selbst zu erarbeiten. Im Prinzip wurde im technischen Bereich der Weg gegangen, den die BAK-Schrift für Forschendes Lernen mittels Begriffs- und Theoriebildung vorgeschlagen hatte: Teilsysteme bilden und diese forschend bearbeiten.

Fazit: Technisch entwickelnde Forschung ist zu einer gängigen Forschungsform für Forschendes Lernen geworden. Sie bleibt aufwendig, da in der Regel entsprechende Werkstätten und Maschinen vorgehalten werden müssen.

8. Gestaltung: Gestaltung als Forschungsform für Forschendes Lernen findet sich vor allem im Bereich Design. Ein Beispiel von vielen ist das Projekt „Care Design“, das sich mit der nutzungsgerechten Gestaltung von Pflegeheimen befasste 
(Funke, 2013). Gestaltung ist grundlegend für die Arbeit in der Architektur. An der Fachhochschule Potsdam wurde ein Undergraduate Research Opportunities Program (UROP) speziell für Studierende der Architektur entwickelt, um selbstorganisierte studentische Forschung zu unterstützen (Albrecht, 2016). Noch eine Anmerkung zum Projektstudium: Nicht jedes Design- oder Kulturarbeit-Projekt oder jede Architektur-Entwurfsübung ist Forschendes Lernen. Die Design-Ausbildung ist weitgehend als Projektstudium organisiert und manche Design-Lehrenden verstehen Projektarbeit per se als Forschendes Lernen. Hierfür müsste jedoch der Gestaltungsprozess wie ein Forschungsprozess organisiert werden (Heidmann, Klose \& Vielhaber. 2011).

Fazit: Die Nutzung von Gestaltung als Forschungsform für Forschung ist nicht schwierig. Voraussetzung ist ein Minimum an Talent, das für Gestaltungsfächer Zugangsvoraussetzung ist - aber nicht auf diese beschränkt sein muss.

\section{$4 \quad$ Schluss}

Diese neue Typologisierung kann für die weitere Ausgestaltung in den Fächern hilfreich sein, zumal die vorgestellte Bewertung eine Idee davon vermittelt, wie leicht oder schwer sich der Zugang für die Studierenden gestaltet. Dies könnte zukünftig in empirischen Erhebungen geprüft und ausdifferenziert werden. Zwei Punkte sind festzuhalten:

- Forschen - auch im Forschenden Lernen - nutzt die ganze Bandbreite von Erkenntnismöglichkeiten. Hierzu gehören nicht zuletzt Forschungsformen, welche die Erkenntnis im Sinne von techne unterstützen, zum Beispiel die Gestaltung.

- Bestimmte Forschungsformen bieten sich besonders für Forschendes Lernen im Bachelorstudium an. Beobachtung/Erhebung sowie Simulation bieten einen Einstieg in die Forschung und leichten Zugang zu Forschendem Lernen insbesondere in Fächern, in denen der Zugang zur Forschung für Studierende nicht so einfach ist (z. B. Theologie oder Mathematik).

Auf den ersten Blick überraschend waren die Schwierigkeiten, die sich mit der Begriffs- und Theoriebildung als Forschungsform für Forschendes Lernen verbinden. Ähnliches scheint für die Hermeneutik zu gelten. Dies wird jedoch verständlich unter dem Aspekt, dass Hermeneutik und Begriffs- und Theoriebildung schon immer den Kern von Wissenschaft ausgemacht haben. Experimente und Beobachtungen mögen noch so verblüffend und aufschlussreich wirken; erst, wenn sich eine 
Theorie formulieren lässt, zum Besipiel eine Gesetzmäßigkeit oder eine generelle Einsicht, wird daraus Wissenschaft (Bunge, 1967). So kann im Zusammenhang mit Forschungsformen und Forschendem Lernen abschließend eine Vermutung aufgestellt werden:

- Die Fähigkeit zur hermeneutischen Interpretation sowie zur Begriffs- und Theoriebildung basiert auf einer Vielzahl von akademischen Erfahrungen, die sich erst im Laufe wissenschaftlicher Auseinandersetzungen ausbilden. Hingegen lassen sich Tätigkeiten wie Beobachten und Erheben sowie (teilweise) computergestützte Simulationen soweit vereinfachen und an Alltagserfahrungen adaptieren, dass hier ein rascherer Einstieg möglich ist, als es von Hermeneutik oder Begriffs- und Theoriebildung erwartet werden kann.

\section{Literatur}

Albrecht, J. \& Ruhl, E. (2018). Räumliche Interventionen. In J. Lehmann \& H. A. Mieg (Hrsg.), Forschendes Lernen (S.200-213). Potsdam: FH Potsdam.

Albrecht, L. (2016). UROP - Einstieg in Forschung. In L. Albrecht, B. Jubin \& I. Mahnke (Hrsg.), Vom Quadrat zum Format: Forschendes Lernen - Lehrende Forschung an der Fach hochschule Potsdam (S.51-53). Potsdam: FH Potsdam.

Alexander, C. W. J. (1964). Notes on the synthesis of form. New York: Oxford University Press. Aristoteles (2007). Die Nikomachische Ethik (griechisch - deutsch, übersetzt von O. Gigon, 2. Aufl.). Düsseldorf: Artemis \& Winkler.

Birresborn, L. (2017). Untersuchungen zur Wirkung von ionisierter Luft auf Bindemittel und Pigmente. Potsdam: FH Potsdam.

Born, S. \& Bor, L. (2018). Projektlabore im Orientierungsstudium. In J. Lehmann \& H. A. Mieg (Hrsg.), Forschendes Lernen (S. 170-185). Potsdam: FH Potsdam.

Bundesassistentenkonferenz (BAK) (1970). Forschendes Lernen - Wissenschaftliches Prüfen (Schriften der BAK, 5. Neudruck) Bielefeld: UVW.

Bunge, M. (1967). Scientific research I: The search for system. Berlin: Springer.

Flyvbjerg, B. (2001). Making social science matter. Cambridge: Cambridge University Press. Freund, S. (2013). Migrationsgeschichte als Experiment des interdisziplinären Forschenden Lernens. In B. Ammann, D. Bartl, S. Cartes \& B. Klose (Hrsg.), InterFlex: Querdenken erwünscht (S. 46-49). Potsdam: FH Potsdam.

Funke, R. (2013). Forschend Lernen in fremden Lebenswelten. In B. Ammann, D. Bartl, S. Cartes \& B. Klose (Hrsg.), InterFlex: Querdenken erwünscht (S.50-52), Potsdam: FH Potsdam.

Heidmann, F., Klose, A. \& Vielhaber, J. (2011). Erlebbar machen von Forschung für Studierende an Fachhochschulen. In W. Benz, J. Kohler \& K. Landfried (Hrsg.), Handbuch Qualität in Studium und Lehre (HQSL) (Griffmarke E 3.4). Berlin: Raabe 
Kaduk, S. \& Lahm, S. (2018). „Decoding the disciplines“. In J. Lehmann \& H. A. Mieg (Hrsg.), Forschendes Lernen (S. 82-95). Potsdam: FH Potsdam.

Kaufmann, M. E., Satilmis, A. \& Mieg, H. A. (Hrsg.) (2018). Forschendes Lernen in den Geisteswissenschaften: Konzepte, Praktiken und Perspektiven hermeneutischer Fächer. Wiesbaden: Springer VS.

Lehmann, J. \& Mieg, H. A. (Hrsg.) (2018). Forschendes Lernen: Ein Praxisbuch. Potsdam: FH Potsdam.

Lippe, M. (2018). Planspiele als methodisches Werkzeug Forschenden Lernens. In J. Lehmann \& H. A. Mieg (Hrsg.), Forschendes Lernen (S. 296-309). Potsdam: FH Potsdam.

Mehling, S., Tienes, F. \& Schuchardt, M. (2018). Angewandtes Lernen durch Service-Learning. In J. Lehmann \& H. A. Mieg (Hrsg.), Forschendes Lernen (S. 326-342). Potsdam: FH Potsdam.

Mieg, H. A. (1993). Computers as experts? On the non-existence of expert systems. Frankfurt: Peter Lang.

Mieg, H. A. \& Dinter, J. (2017). Forschen im Forschenden Lernen: Der Einfluss von Forschungsform, Erkenntnisinteresse und Praxiskooperation. In H. Laitko, H. A. Mieg \& H. Parthey (Hrsg.), Forschendes Lernen: Wissenschaftsforschung Jahrbuch 2016 (S.29-50). Berlin: wvb.

Mieg, H. A. \& Lehmann, J. (Hrsg.) (2017). Forschendes Lernen: Wie die Lehre in Universität und Fachhochschule erneuert werden kann. Frankfurt: Campus.

Mieg, H. A., de Sombre, S. \& Naef, M. A. (2013). How formality works: The case of environmental professionals. Professions \& Professionalism, 3(1). Verfügbar unter https:// journals.hioa.no/index.php/pp/article/view/564/1443 [06.06.2019].

Monö, R. (1997). Design for product understanding: The aesthetics of design from a semiotic approach. Stockholm: Liber.

Müller-Christ, G. (2018). Wie kommt das Neue in die Welt? Forschungsorientierte Lehre in der Betriebswirtschaftslehre mit Systemaufstellungen. In J. Lehmann \& H. A. Mieg (Hrsg.), Forschendes Lernen (S.96-108). Potsdam: FH Potsdam.

Pace, D. \& Middendorf, J. (Hrsg.) (2004). Decoding the disciplines: Helping students learn disciplinary ways of thinking. San Francisco: Jossey-Bass.

Prytula, M. \& Schröder, T. (Hrsg.) (2018). Städtische Transformationsprozesse. Potsdam: FH Potsdam.

Prytula, M., Schröder, T. \& Mieg, H. A. (2017). Inter- und Transdisziplinarität. In H. A. Mieg \& J. Lehmann, J. (Hrsg.), Forschendes Lernen (S. 138-147). Frankfurt: Campus.

Radermacher, M., Runge, K. \& Scherfner, M. (2018). „Innovationsmodul Bremssysteme“ ein Konzept zum Forschenden Lernen in technischen Disziplinen. In J. Lehmann \& H. A. Mieg (Hrsg.), Forschendes Lernen (S.344-349). Potsdam: FH Potsdam.

Reinmann, G. (2018). Lernen durch Forschung - aber welche? In N. Neuber, W. Paravicini \& M. Stein (Hrsg.), Forschendes Lernen - the wider view. Münster: WTM.

Ritter, J. \& Gründer, K. (1998). Technik. In J. Ritter \& K. Gründer (Hrsg.), Historisches Wörterbuch der Philosophie (Band 10, S.940-952). Darmstadt: Wissenschaftliche Buchgesellschaft.

Rubel, K. (2017). Forschungsprojekt „Lernen“. In H. Laitko, H. A. Mieg \& H. Parthey (Hrsg.), Forschendes Lernen (S. 83-98). Berlin: wvb.

Sagasti, F. (2000). The twilight of the Baconian age and the future of humanity. Futures, 32, S. 595-602. 
Schmidt-Wenzel, A. \& Rubel, K. (2018). Forschungsgeleitete Lehre. In M. E. Kaufmann, A. Satilmis \& H. A. Mieg (Hrsg.), Forschendes Lernen in den Geisteswissenschaften: Konzepte, Praktiken und Perspektiven hermeneutischer Fächer (S. 191-210). Wiesbaden: Springer VS.

Schwanewedel, J., Großschedl, J. \& Heyduck, B. (2018). Forschend Lehren lernen: Lehramtsstudierende betreiben Bildungsforschung. In J. Lehmann \& H. A. Mieg (Hrsg.), Forschendes Lernen (S. 138-151). Potsdam: FH Potsdam.

Selje-Aßmann, N., Poll, C., Tisler, M., Gerstenberg, J., Blum, M. \& Fleischer, J. (2017). Forschendes Lernen in den Lebenswissenschaften. In H. A. Mieg \& J. Lehmann (Hrsg.), Forschendes Lernen (S.200-210). Frankfurt: Campus.

Wissenschaftsrat (2012). Empfehlungen zur Weiterentwicklung der wissenschaftlichen Informationsinfrastrukturen in Deutschland bis 2020. Berlin: Wissenschaftsrat. Verfügbar unter https://www.wissenschaftsrat.de/download/archiv/2359-12.pdf [06.06.2019].

Wright, G. H. von (1984). Erklären und Verstehen (2. Aufl.). Königstein: Athenäum (Original 1971: Explanation and Understanding).

\section{Zum Autor}

Prof. Dr. Harald A. Mieg ist (Honorar-)Professor für Metropolen- und Innovationsforschung an der Humboldt-Universität zu Berlin. Seine Forschungsbereiche sind Stadtentwicklungsplanung, Hochschulforschung und Professionalisierung. Von 2014 bis 2018 leitete er das nationale Verbundforschungsprojekt „ForschenLernen“. E-Mail: harald.mieg@hu-berlin.de

Open Access Dieses Kapitel wird unter der Creative Commons Namensnennung 4.0 International Lizenz (http://creativecommons.org/licenses/by/4.0/deed.de) veröffentlicht, welche die Nutzung, Vervielfältigung, Bearbeitung, Verbreitung und Wiedergabe in jeglichem Medium und Format erlaubt, sofern Sie den/die ursprünglichen Autor(en) und die Quelle ordnungsgemäß nennen, einen Link zur Creative Commons Lizenz beifügen und angeben, ob Änderungen vorgenommen wurden.

Die in diesem Kapitel enthaltenen Bilder und sonstiges Drittmaterial unterliegen ebenfalls der genannten Creative Commons Lizenz, sofern sich aus der Abbildungslegende nichts anderes ergibt. Sofern das betreffende Material nicht unter der genannten Creative Commons Lizenz steht und die betreffende Handlung nicht nach gesetzlichen Vorschriften erlaubt ist, ist für die oben aufgeführten Weiterverwendungen des Materials die Einwilligung des jeweiligen Rechteinhabers einzuholen.

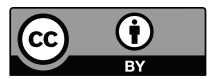

